\title{
Holographic Reconstruction of Finite Airy Beams with Self-Healed and Multiplexed Features
}

\author{
Dawoon Choi ${ }^{1}$, Keehoon Hong ${ }^{1}$, Kyoung-Youm Kim², Kyookeun Lee ${ }^{1}$, \\ Il-Min Lee ${ }^{1}$, and Byoungho Lee ${ }^{1}$ * \\ ${ }^{I}$ National Creative Research Center for Active Plasmonics Application Systems, \\ Inter-University Semiconductor Research Center and \\ School of Electrical Engineering, Seoul National University, \\ Seoul 151-744, Korea \\ ${ }^{2}$ Department of Optical Engineering, Sejong University, Seoul 143-741, Korea
}

(Received August 13, 2014 : revised October 6, 2014 : accepted October 27, 2014)

\begin{abstract}
To generate finite-power Airy beams, a novel holographic method is proposed. We record the interference pattern between an Airy beam (signal beam) and plane wave (reference beam) on a photopolymer, then decode the hologram by illuminating with the reference beam. The reconstructed beams still present the non-diffraction, acceleration, and self-healing features of optical Airy beams. In addition, angular multiplexing of two Airy beams with opposite acceleration directions is presented.
\end{abstract}

Keywords: Finite Airy beam, Holography, Angular multiplexing

OCIS codes : (260.2110) Electromagnetic optics; (070.2580) Paraxial wave optics; (090.4220) Multiplex holography

\section{INTRODUCTION}

An Airy beam is a nonspreading wave that propagates along a ballistic trajectory without any external force. Since Berry and Balazs illuminated this strange characteristic of the Airy wave packet in quantum mechanics [1], interest in Airy beams has steadily increased [2-18].

Although it is impossible to implement ideal Airy beams because they carry infinite power, so-called finite Airy beams can be achieved by tailoring infinite side lobes with an aperture function; these finite beams have propagation characteristics similar to those of ideal Airy beams, as was first shown by Siviloglou and Christodoulides [2]. Based on their report, the first optical observation of Airy beams was realized by an optical Fourier-transform system that imposed cubic phase on a broad Gaussian beam [3]. According to the same principle, nonlinear generation of Airy beams [4], curved plasma channel generation [5], and electron-beam generation [6] were reported. Another way to generate a finite Airy beam, which directly assigns the initial launching conditions, was also suggested [7]. By matching initial intensity and phase distributions of Airy beams with those of surface plasmon polaritons (SPPs) after passing through metal slits or gratings, (1+1)-dimensional $((1+1) \mathrm{D})$ Airy beams can be launched. Also, an Airy beam generated in free space can be coupled via periodic gratings on a metal surface and dynamically controlled by changing the position of either the objective lens or phase mask [8].

In this paper, we propose holographic generation of finite Airy beams by "reading" a hologram that is recorded as the interference between a finite Airy beam, generated by optical Fourier transform, and a reference plane wave [9]. Although bulk optical components are necessary in the initial recording process, once the initial recording is over we can generate finite Airy beams using only a simple plane wave. Moreover, this method can exploit the unique features of holography itself, such as successful reconstruction even with imperfect incidence of the reference beam, and multiplexing of several Airy beams.

This paper is organized as follows. After a brief introduction to Airy beams in Section II, in Section III we show that the holographically generated beam maintains non-diffracting nature with a bending trajectory, as well as self-healing. Finally, in Section IV, using the characteristics of holography,

\footnotetext{
*Corresponding author: byoungho@snu.ac.kr

Color versions of one or more of the figures in this paper are available online.
} 
we present angular multiplexing of two Airy beams determined by the angles of reference beams.

\section{THEORETICAL ANALYSIS OF AIRY BEAMS}

Let us invoke the solution of a (1+1)D Airy beam from the paraxial Helmholtz equation with the potential-free condition:

$$
i \frac{\partial \phi}{\partial \xi}+\frac{1}{2} \frac{\partial^{2} \phi}{\partial s^{2}}=0
$$

where $\phi$ denotes the optical field, and $s\left(=x / x_{0}\right.$, with $x_{0}$ being an arbitrary scaling factor) and $\xi\left(=z / k x_{0}^{2}, k\right.$ being the wavenumber in a propagating medium) represent the transverse and longitudinal coordinates respectively. By solving Eq. (1), an ideal Airy-beam solution is obtained [2]:

$$
\phi(s, \xi)=\operatorname{Ai}\left(s-\left(\frac{\xi}{2}\right)^{2}\right) \exp \left(i\left(\frac{s \xi}{2}\right)-i\left(\frac{\xi^{3}}{12}\right)\right) .
$$

Since Eq. (2) is not square-integrable, an aperture function $\exp (a s)$ is necessary to tailor infinite side lobes for experimental realization of Airy beams. Thus at the origin $(z=0)$ the form of the Airy beam is given by

$$
\phi(s, \xi=0)=\operatorname{Ai}(s) \exp (a s)
$$

where $a$ is positive. It is clear that the more $a$ deviates from zero, the more the energy of this beam (called a finite Airy beam) is confined, while the beam becomes analogous to an ideal Airy beam as $a$ approaches zero [10].

In the case of a $(2+1)$-dimensional $((2+1) \mathrm{D})$ finite Airy beam, Eq. (3) can be expanded to

$$
\phi(x, y, z=0)=\operatorname{Ai}\left(\frac{x}{x_{0}}\right) \exp \left(a \frac{x}{x_{0}}\right) \operatorname{Ai}\left(\frac{y}{y_{0}}\right) \exp \left(a \frac{y}{y_{0}}\right),
$$

where $x_{0}$ and $y_{0}$ are arbitrary scaling factors along the $x$ and $y$ coordinates respectively. Throughout this paper, the parameters of the finite Airy beams $\left(x_{0}, y_{0}\right.$, and $\left.a\right)$ are set to $50 \mu \mathrm{m}, 50 \mu \mathrm{m}$, and 0.1 respectively, and a wavelength of $633 \mathrm{~nm}$ is assumed. We can see the intensity distributions of the $(2+1) \mathrm{D}$ finite Airy beam with these parameters at positions $z=0, z=5 \mathrm{~cm}$, and $z=15 \mathrm{~cm}$ in Figs. 1(a), 1(b), and 1(c), respectively.

\section{HOLOGRAPHIC RECORDING AND RECONSTRUCTION OF AIRY BEAMS}

Holography is a technique that can record and reconstruct both the amplitude and the phase of an optical wave [19]. Figure 2 is a schematic diagram of the recording procedure for holographic generation of an Airy beam. After passing through expanding optics and a beam splitter 1 (BS1), a plane wave from a He-Ne laser (Research Electro-Optics, LSRP-3501) with wavelength $633 \mathrm{~nm}$ is divided into signal and reference arms. The signal arm is consisted of a $2-f$

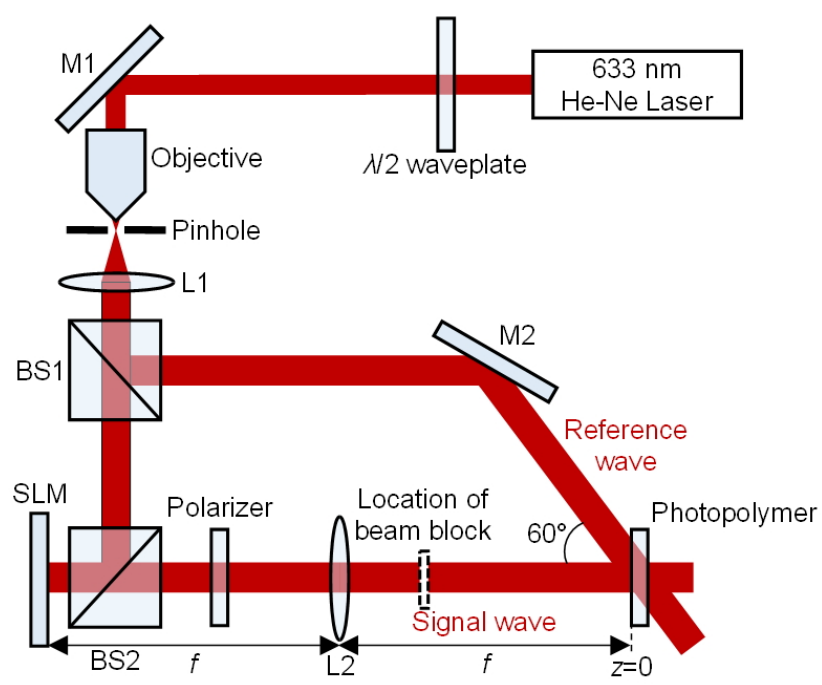

FIG. 2. Schematic diagram of the recording and reconstruction procedures for the holographic generation of an Airy beam.

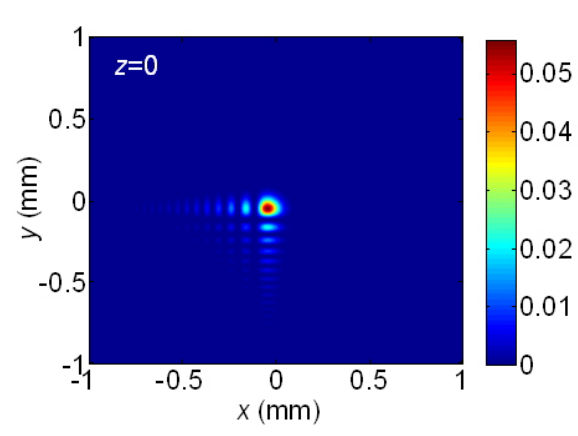

(a)

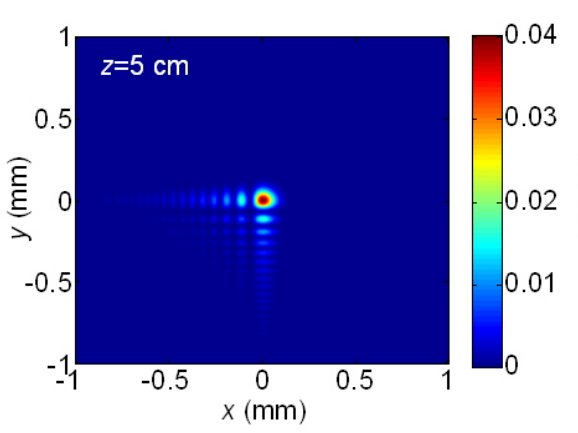

(b)

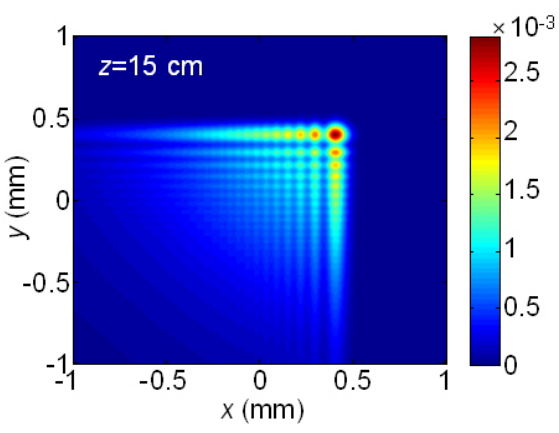

(c)

FIG. 1. Intensity distributions of a (2+1)D finite Airy beam at (a) $z=0$, (b) $z=5 \mathrm{~cm}$, and (c) $z=15 \mathrm{~cm}$. 
system: a spatial light modulator (SLM) (Holoeye Pluto, reflection type, $1920 \times 1080$ pixels, pixel pitch $8 \mu \mathrm{m}$ ) that imposes cubic phase, and lens 2 (L2) with a focal length $f$ of $50 \mathrm{~cm}[20]$. We place L2 at a distance $f$ from the SLM to perform the optical Fourier transform. A $\lambda / 2$ wave plate and linear polarizer are used to adjust the polarization states and obtain clear images respectively. After the signal beam is reflected from the SLM and travels $f$ after passing through L2, i.e. at the Fourier plane (which is taken as $z=0$ ), a finite Airy beam is obtained.

Before actually recording, we confirm that the signal beam is the desired finite Airy beam using a charge-coupled device (CCD). We use a first-order diffracted beam from the SLM for Airy beam generation and hologram recording, to avoid the DC term in the zeroth-order diffracted Airy beam. The captured images (without photopolymer) of a $(2+1) \mathrm{D}$ finite Airy beam at $z=0$ (at the Fourier plane), $z=5 \mathrm{~cm}$, and $z=15 \mathrm{~cm}$ are shown in Figs. 3(a), 3(b), and 3(c), respectively. After this, interference patterns between signal and reference (a plane wave) beams are recorded on photopolymer film [21]. In this procedure, the reference beam has an incident angle of $60^{\circ}$ with an exposed energy of 32 $\mathrm{mJ} / \mathrm{cm}^{2}$. The photopolymer film, which is laminated on a slide glass (refractive index 1.518) consists of two layers: a photopolymer layer of thickness ranging from 14 to $18 \mu \mathrm{m}$ and optically clear plastic substrate layer (refractive index 1.57) of $175 \mu \mathrm{m}$ thickness. The refractive index of the photopolymer layer before the hologram recording is 1.485 , and the maximum value of refractive index modulation is
0.03 with $633-\mathrm{nm}$ illumination. We select this material because it is easy to handle, and the post-exposure processing is quite simple. For curing, the recorded sample is exposed to a UV lamp with a power density of $110 \mathrm{~mW} / \mathrm{cm}^{2}$ for 3 minutes, the distance between lamp and sample being kept to about $30 \mathrm{~cm}$.

To reconstruct the Airy beam, we place a beam blocker (indicated as a dotted box in Fig. 2) in the signal-beam path, to shine the reference beam without a signal beam. As a result, the signal beam, i.e. the $(2+1) \mathrm{D}$ finite Airy beam, is simply generated from the photopolymer without any SLM or lens optics. Captured intensity distributions of the reconstructed beam at $z=5 \mathrm{~cm}$ and $z=15 \mathrm{~cm}$ are shown in Figs. 3(d) and 3(e) respectively. Here we cannot present the intensity pattern of the reconstructed beam at $z=0$, because the CCD camera cannot be placed in front of the photopolymer surface. Comparing Figs. 3(d) and 3(e) to Figs. 1(b) and 1(c), or Figs. 3(b) and 3(c), we can see that the reconstructed beam shows intensity distributions similar not only to those of the calculated Airy beam, but also to those of the SLM-generated (signal) Airy beam. In addition, after propagating $15 \mathrm{~cm}$ in the $+z$-direction they still coincide well, as shown in Figs. 1(c), 3(c), and 3(e). These results show that the reconstructed beam is a holographically generated Airy beam retaining the same characteristics of the original Airy beam, such as acceleration and non-diffraction.

To provide a quantitative analysis of the propagation behaviors of SLM-generated Airy beams and reconstructed Airy beams, cross-correlations with the ideal Airy beam

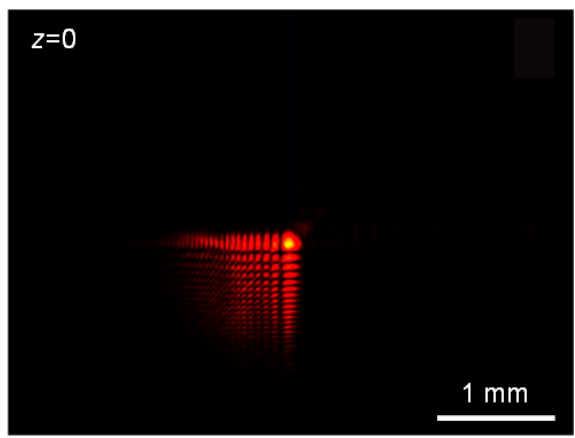

(a)

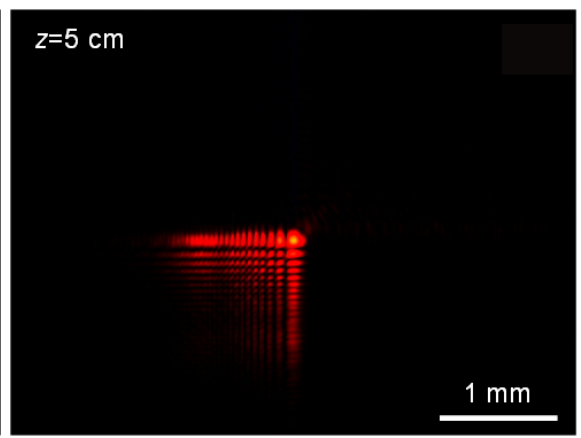

(b)

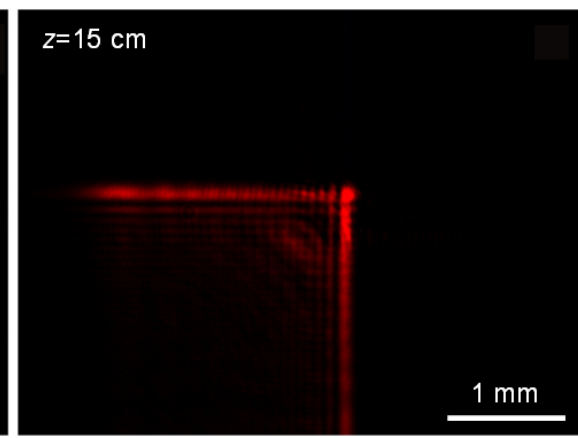

(c)

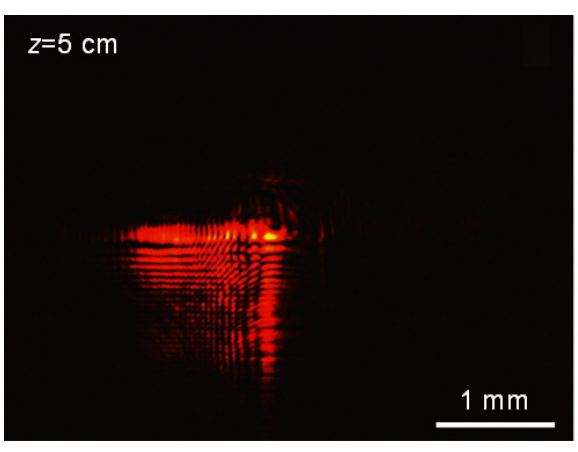

(d)

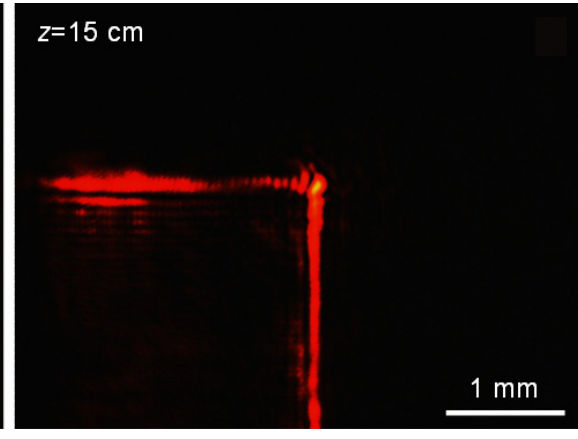

(e)

FIG. 3. CCD images of the signal beam (a (2+1)D finite Airy beam) at (a) $z=0$, (b) $z=5 \mathrm{~cm}$, and (c) $z=15 \mathrm{~cm}$, plus CCD images of the holographically reconstructed Airy beam at (d) $z=5 \mathrm{~cm}$ and (e) $z=15 \mathrm{~cm}$. 
are calculated from the cross-sections of the main lobes along the $x$-direction at $z=5 \mathrm{~cm}$ and $z=15 \mathrm{~cm}$, respectively. We define the maximum value of the cross correlation $C_{1}(z)$ as follow:

$$
C_{1}(z)=\max \left(\int_{-\infty}^{+\infty}\left|\phi_{0}^{*}(\tau)\right|^{2}\left|\phi_{i=1,2}(x+\tau)\right|^{2} d \tau\right)
$$

where $\phi_{0}$ is the ideal Airy beam, $\phi_{1}$ is the SLM generated Airy beam, and $\phi_{2}$ is the reconstructed Airy beam. When $C_{1}(z)$ between the ideal Airy beam and the reconstructed Airy beam is normalized to that of the SLM-generated Airy beam, the values of $C_{1}(z)$ are $0.79(z=5 \mathrm{~cm})$ and 0.63 $(z=15 \mathrm{~cm})$ respectively.

As another confirmation, we can check whether the holographically reconstructed beam has the self-healing feature [11], a distinguishing property of various types of diffractionfree beams. Here we consider two cases of self-healing in the reconstruction procedure. First, an opaque obstacle is placed between the photopolymer and the $z=5 \mathrm{~cm}$ plane, to block the main lobe of the reconstructed beam. The location of the obstacle is indicated in Fig. 4(a) as a white box. This veiled main lobe is, however, reconstructed after the propagation distance of $15 \mathrm{~cm}$, as indicated by the white circle in Fig. 4(c). That is, although the main lobe of the generated beam is blocked, its original shape can be reconstructed during propagation; thus the holographically generated Airy beam has the self-healing characteristic, as expected.

As a second case, we partially block the reference beam with an obstacle placed between M2 and the photopolymer, to achieve partial illumination of the recorded medium. The CCD image of the reconstructed beam at $z=5 \mathrm{~cm}$ is shown in Fig. 5(a). Similar to the first case shown in Fig. 4, after propagating 10 or $15 \mathrm{~cm}$ the reconstructed beam recovers its original Airy shape, as can be seen in Fig. 5(c), indicated as a white circle. Although the self-healed Airy beams in both Figs. 4 and 5 have decreased energy due to the beam blocking, the results show that the main lobes of the holographically generated Airy beams are recovered. This means that robust reconstruction of the original information (self-healing) can be realized, even if significantly imperfect incidence occurs for the reference beam.

\section{ANGULAR MULTIPLEXING OF AIRY BEAMS}

Finally, we investigate the feasibility of angular multiplexing

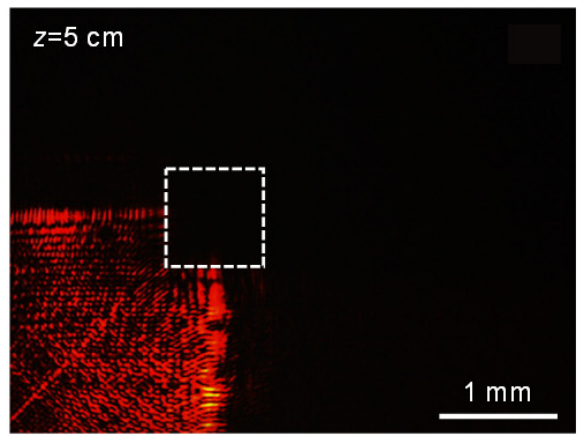

(a)

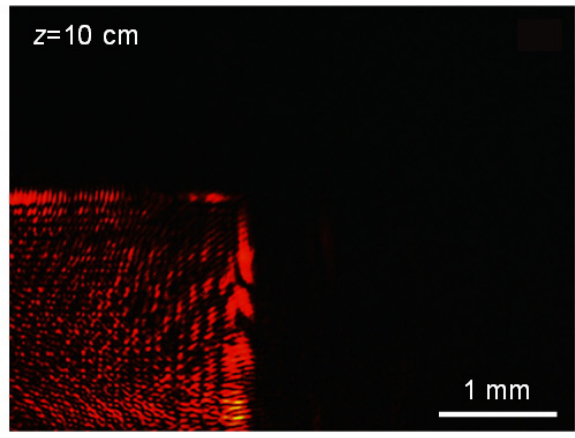

(b)

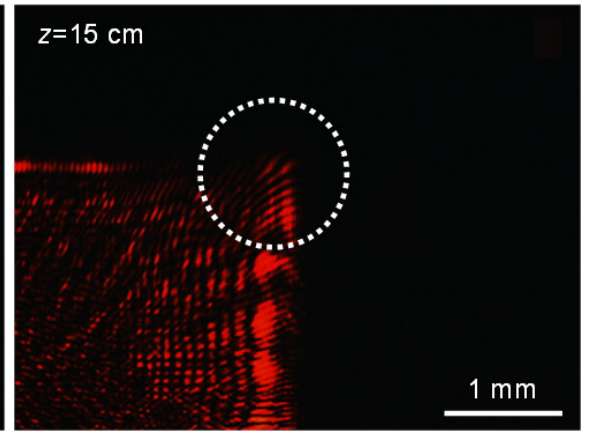

(c)

FIG. 4. (a) A holographically generated Airy beam that is blocked at $z=5 \mathrm{~cm}$. Its intensity patterns (CCD images) at (b) $z=10 \mathrm{~cm}$ and (c) $z=15 \mathrm{~cm}$ clearly demonstrate the self-healing characteristic of a holographically generated beam.

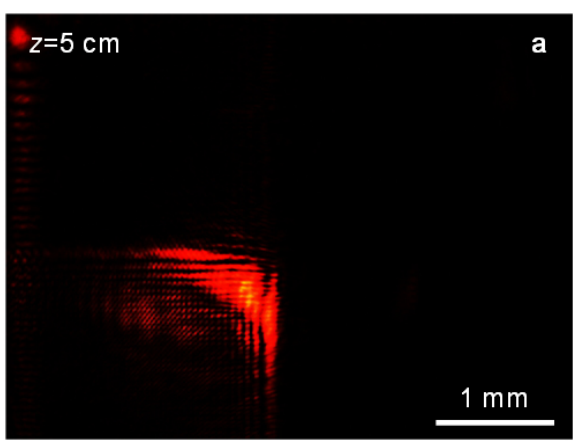

(a)

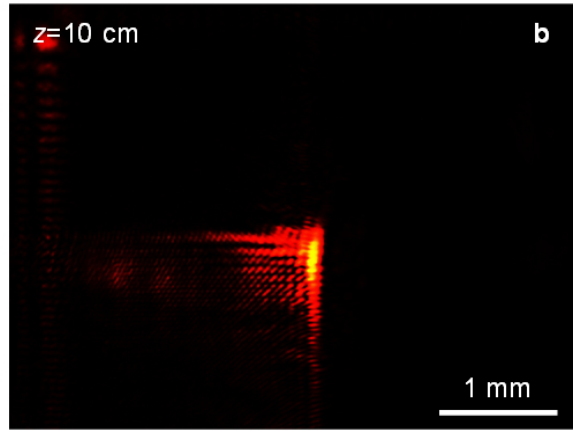

(b)

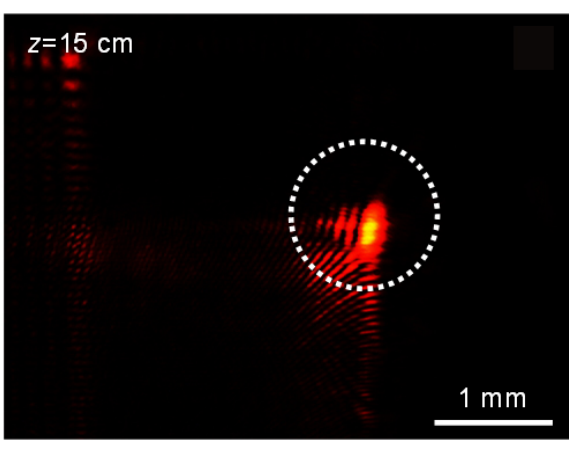

(c)

FIG. 5. Beam reconstruction for the case of partial incidence of the reference beam. Profiles of reconstructed beams captured at (a) $z=5 \mathrm{~cm}$, (b) $z=10 \mathrm{~cm}$, and (c) $z=15 \mathrm{~cm}$. The white circle denotes the main lobe (veiled) region. 
of Airy beams based on holography. In this case the recording procedure is conducted twice, with two reference beams at different incidence angles. In Fig. 6 the experimental setup for Airy beam multiplexing is presented. Two reference beams are incident to the photopolymer with $60^{\circ}$ and $-60^{\circ}$ incidence angles respectively, and energy density of 32 $\mathrm{mJ} / \mathrm{cm}^{2}$ is dosed as in the previous section. To compensate for the power difference between two reference beams, a

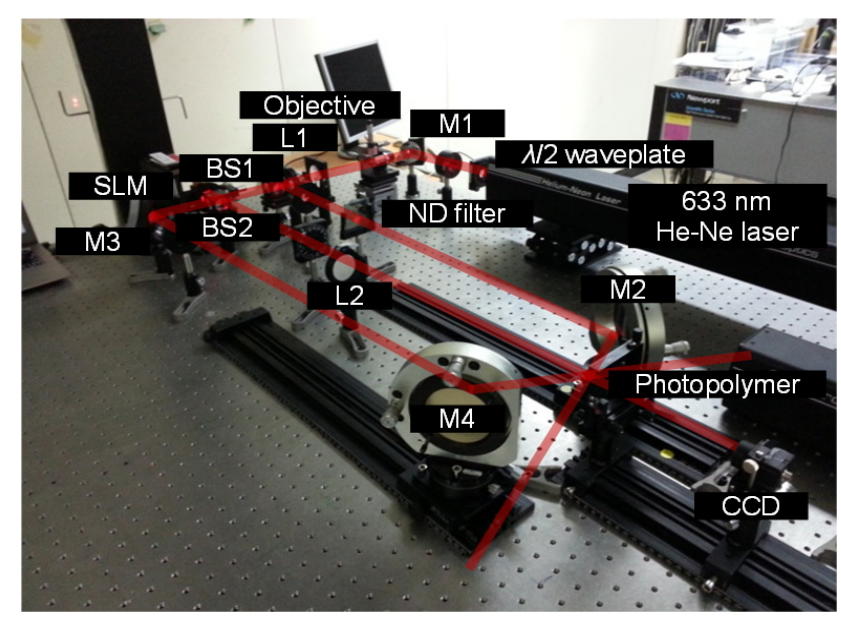

FIG. 6. Experimental setup for Airy-beam multiplexing (M: mirror, L: lens, BS: beam splitter).

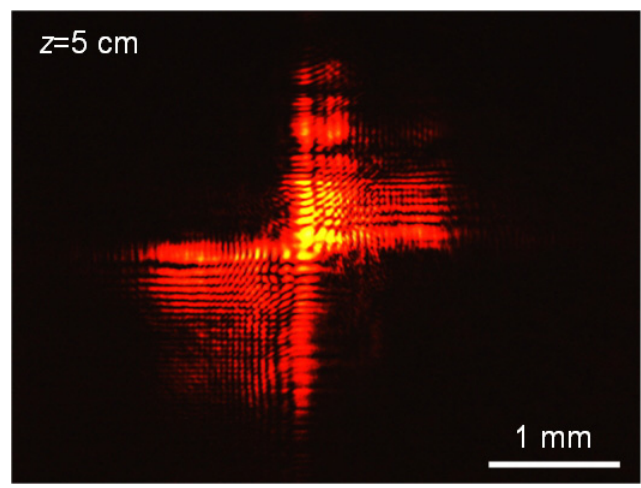

(a)

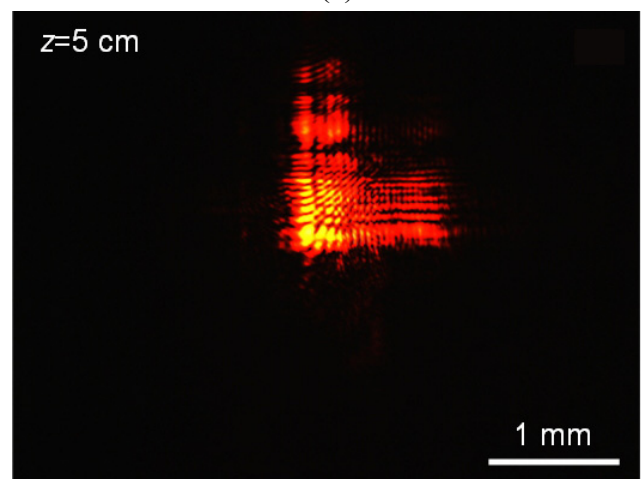

(c) neutral density (ND) filter is located in reference arm 1. Here reference arms 1 and 2 pass through M2 and M4 respectively. First, a $(2+1) \mathrm{D}$ finite Airy beam with $x_{0}=50$ $\mu \mathrm{m}$ and $y_{0}=50 \mu \mathrm{m}$ is recorded on the photopolymer, while opening reference arm 1 and blocking reference arm 2 . Next, another $(2+1) \mathrm{D}$ finite Airy beam with $x_{0}=-50 \mu \mathrm{m}$ and $y_{0}=-50 \mu \mathrm{m}$ is recorded in the same area of the photopolymer, while opening only reference arm 2 . Each Airy beam propagates with a different acceleration direction when both signal Airy beams are successively recorded on the same area of the photopolymer with the respective reference beams.

To reconstruct recorded Airy beams, we place an additional barrier between lens and photopolymer, to block the signal beam. After that, two reference beams are incident upon the photopolymer at the same time, and we obtain two Airy beams accelerating in opposite directions, as shown in Figs. 7(a) and 7(b), which are captured by the CCD at $z=5 \mathrm{~cm}$ and $z=15 \mathrm{~cm}$ respectively. Moreover, when we block only reference arm 1 we can reconstruct only one Airy beam, as shown in Figs. 3(d) and 3(e), which are captured at $z=5 \mathrm{~cm}$ (Fig. 7(c)) and $z=15 \mathrm{~cm}$ (Fig. 7(d)) respectively. That is, each Airy beam can be individually controlled via appropriate blocking of a reference beam. This can be utilized for on/off switching or routing in optical signal processing. Also, it can be applied to particle manipulation: a portion of particles can be held or released while others are released or held, respectively.

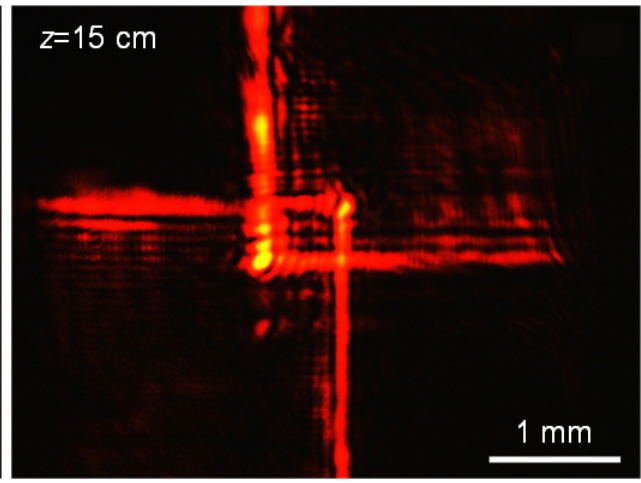

(b)

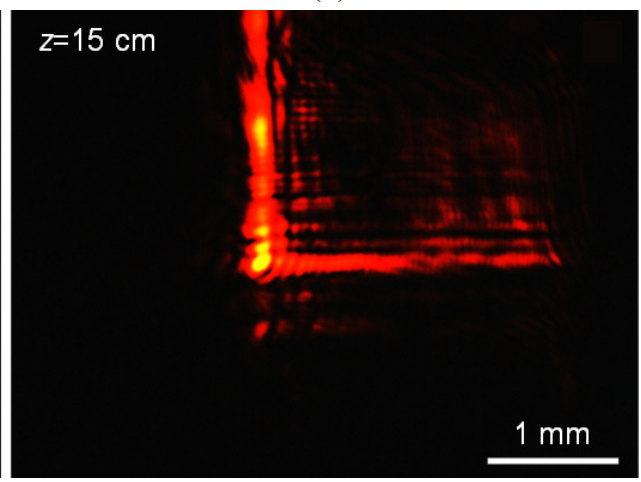

(d)

FIG. 7. CCD images of reconstructed multiple Airy beams at (a) $z=5 \mathrm{~cm}$ and (b) $z=15 \mathrm{~cm}$, plus reconstructed Airy beams at (c) $z=5$ $\mathrm{cm}$ and (d) $z=15 \mathrm{~cm}$ when reference beam 1 is blocked. 


\section{CONCLUSION}

In this paper, a novel Airy beam generation method based on holography is presented. The proposed method has the advantage that once holograms are recorded in the photopolymer, bulky optics such as SLM and lenses are not needed to generate Airy beams. In addition, multiple Airy beams can be stored and reconstructed simultaneously or individually. We hope that these results find practical uses, such as in particle manipulation or optical signal processing [14-18].

\section{ACKNOWLEDGMENT}

This work was supported by the National Research Foundation of Korea grant, funded by the Korean government (MSIP) through the Creative Research Initiatives Program (20070054847). The authors acknowledge support from Bayer MaterialScience AG in providing the photopolymer Bayfol ${ }^{\mathbb{R}}$ HX film.

\section{REFERENCES}

1. M. Berry and N. Balazs, "Nonspreading wave packets," Am. J. Phys. 47, 264-267 (1979).

2. G. A. Siviloglou and D. N. Christodoulides, "Accelerating finite energy Airy beams," Opt. Lett. 32, 979-981 (2007).

3. G. A. Siviloglou, J. Broky, A. Dogariu, and D. N. Christodoulides, "Observation of accelerating Airy beams," Phys. Rev. Lett. 99, 213901 (2007).

4. T. Ellenbogen, N. Voloch-Bloch, A. Ganany-Padowicz, and A. Arie, "Nonlinear generation and manipulation of Airy beams," Nat. Photon. 3, 395-398 (2009).

5. P. Polynkin, M. Kolesik, J. V. Moloney, G. A. Siviloglou, and D. N. Christodoulides, "Curved plasma channel generation using ultraintense Airy beams," Science 324, 229-232 (2009).

6. N. Voloch-Bloch, Y. Lereah, Y. Lilach, A. Gover, and A. Arie, "Generation of electron Airy beams," Nature 494, 331-335 (2013).

7. D. Choi, Y. Lim, I.-M. Lee, S. Roh, and B. Lee, "Airy beam excitation using a subwavelength metallic slit array,"
IEEE Photon. Technol. Lett. 24, 1440-1442 (2012).

8. P. Zhang, S. Wang, Y. Liu, X. Yin, C. Lu, Z. Chen, and $\mathrm{X}$. Zhang, "Plasmonic Airy beams with dynamically controlled trajectories," Opt. lett. 36, 3191-3193 (2011).

9. D. Choi, K. Hong, K. Lee, J. Cho, I.-M. Lee, and B. Lee, "Airy beam generation based on holographic recording and reconstruction," in Proc. Digital Holography and ThreeDimensional Imaging, OSA Technical Digest (Kohala Coast, Hawaii, USA, 2013), paper DW2A21.

10. D. Choi, K. Lee, K. Hong, I.-M. Lee, K.-Y. Kim, and B. Lee, "Generation of finite power Airy beams via initial field modulation," Opt. Express 21, 18797-18804 (2013).

11. J. Broky, G. A. Siviloglou, A. Dogariu, and D. N. Christodoulides, "Self-healing properties of optical Airy beams," Opt. Express 16, 12880-12891 (2008).

12. G. A. Siviloglou, J. Broky, A. Dogariu, and D. N. Christodoulides, "Ballistic dynamics of Airy beams," Opt. Lett. 33, 207-209 (2008).

13. C.-Y. Hwang, D. Choi, K.-Y. Kim, and B. Lee, "Dual Airy beam," Opt. Express 18, 23504-23516 (2010).

14. J. Baumgartl, M. Mazilu, and K. Dholakia, "Optically mediated particle clearing using Airy wavepackets," Nat. Photon. 2, 675-678 (2008).

15. D. N. Christodoulides, "Optical trapping: Riding along an Airy beam," Nat. Photon. 2, 652-653 (2008).

16. P. Zhang, J. Prakash, Z. Zhang, M. S. Mills, N. K. Efremidis, D. N. Christodoulides, and Z. Chen, "Trapping and guiding microparticles with morphing autofocusing Airy beams," Opt. Lett. 36, 2883-2885 (2011).

17. Z. Zheng, B.-F. Zhang, H. Chen, J. Ding, and H.-T. Wang, "Optical trapping with focused Airy beams," Appl. Opt. 50, 43-49 (2011).

18. P. Rose, F. Diebel, M. Boguslawski, and C. Denz, "Airy beam induced optical routing," Appl. Phys. Lett. 102, 101101 (2013).

19. J. Goodman, Introduction to Fourier Optics, 3rd ed. (Roberts \& Company, 2005).

20. T. Khos-Ochir, P. Munkhbaatar, B. Yang, H. Kim, J. Kim, and M. Kim, "Polarimetric measurement of Jones matrix of a twisted nematic liquid crystal spatial light modulator," J. Opt. Soc. Korea 16, 443-448 (2012).

21. K Lee, S. Jeung, B. Cho, and N. Kim, "Photopolymer-based surface-normal input/output volume holographic grating coupler for 1550-nm optical wavelength," J. Opt. Soc. Korea 16, 17-21 (2012). 